\title{
Rejuvenecimiento periorbitario no invasivo
}

\section{Non-invasive periorbital rejuvenation}

\author{
Muñóz del Olmo, J . L.*, Serra Renom, J . M.**
}

Resumen

Cirujanos plásticos y médicos estéticos se esfuerzan por lograr resultados satisfactorios y estéticos que mejoren o suavicen el paso del tiempo a nivel facial, con un especial interés o énfasis en el área periorbitaria.

Un gran número de pacientes consultan para mejorar esta zona, pero por diferentes motivos desean que los procedimientos que se les realicen sean poco invasivos y con resultados rápidos, permitiéndoles así incorporarse lo antes posible a sus actividades cotidianas.

Es fundamental el conocimiento de las proporciones faciales y periorbitarias para lograr resultados naturales.

El objetivo de la técnica que proponemos es lograr una bioestimulación local de la piel, restaurar los volúmenes y reducir las arrugas de expresión o dinámicas, consiguiendo así una apariencia relajada y juvenil en el paciente.

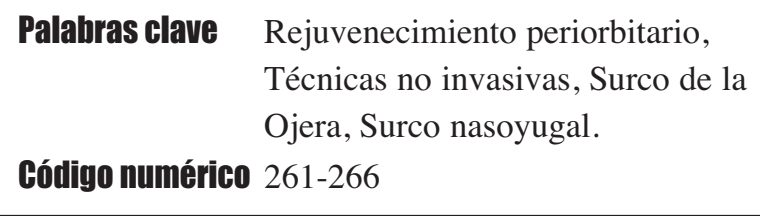

Código numerico 261-266

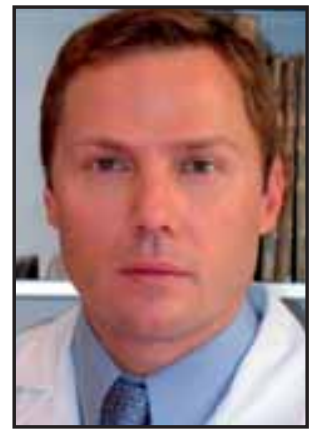

Muñoz del Olmo, J , L.
Plastic surgeons and aesthetic doctors are making an effort to reach positive aesthetic results. Their aim is to soften the effects of age on facial features stressing in the periorbital area.

Many patients come to improve their facial image on this area, but they are asking for non-invasive and fast procedures to keep on their daily life.

It is indispensable the knowledge of facial and periorbital proportions to achieve a more natural effect.

The aim of the technique exposed is to achieve a local bioestimulation on skin, to restore volumes and to reduce expression and dynamical wrinkles. The expected result is a relaxing, youthful appearance.

$\begin{array}{ll}\text { Key words } & \begin{array}{l}\text { Periorbital Rejuvenation, Non-invasive } \\ \text { techniques, Eye bag wrinkle, } \\ \text { Tear-through }\end{array} \\ \text { Numeral Code } & 261-266\end{array}$




\section{Introducción}

La mirada y su entorno, son los puntos focales de todo el rostro, donde se sintetizan las emociones, los estados de ánimo y los signos del paso del tiempo. Por estas razones siempre ha existido un especial interés en mantener este área joven y hermosa, tanto en mujeres como en hombres, a todas las edades.

Hoy en día existe un gran número de procedimientos quirúrgicos para rejuvenecer y embellecer la mirada y su entorno (blefaroplastia, elevación de cejas con dispositivos reabsorbibles, cantopexia, lifting temporal endoscópico, corrección del surco de la ojera con reposición de bolsas grasas o con implantación de grasa o colocación de materiales prótesicos). Sin embargo, tenemos pacientes que no ameritan procedimientos tan invasivos por ser muy jóvenes o bien por otras circunstancias, o que no desean o no pueden someterse a cirugía (miedo, contraindicación quirúrgica etc.).

En la actualidad el campo de la Medicina Estética cuenta con importantes avances, seguros y con muy buenos resultados. Por esto el cirujano plástico con interés en el campo de la Cirugía Estética, tiene un gran aliado en estos procedimientos médico-estéticos, pues complementan y realzan (sin sustituir) la cirugía de la mirada.

Por estas razones, cirujanos plásticos de diferentes países se han decidido a incluir procedimientos no invasivos como parte de su arsenal terapéutico a la hora de rejuvenecer las diferentes subunidades del rostro; entre ellos destacan los doctores Philipe Levy, Mauricio De Maio y Gerardo Manuell Lee, entre otros. La unión y combinación de todas estas técnicas no invasivas ya bien conocidas tanto por cirujanos plásticos como por médicos estéticos es sintetizada y protocolizada por el Dr. Manuell Lee con el nombre de Mesoplastia; definiéndola como una terapia específica antienvejecimiento que utiliza agujas intradérmicas para estimular los procesos metabólicos del organismo y lograr una reestructuración cutánea, consiguiendo un rejuvenecimiento de las diferentes áreas faciales sin cirugía.

Como siempre, debemos tener un adecuado conocimiento antropométrico y anatómico de las subunidades faciales y de las proporciones de las mismas entre si. Numerosos estudios (Langlois y Roggman, Baudouin y Tiberghien), nos orientan sobre la correcta y armónica situación de cejas, párpado superior e inferior, canto externo, área infraorbitaria, etc. El Dr. Oscar Ramírez en su artículo sobre "El ojo hermoso" realiza un excelente análisis y síntesis de todos estos estudios (1).

Cuando un paciente llega a la consulta y nos explica qué áreas de su rostro quiere mejorar, debemos
PARAMETROS DE EMVEJECIMMENTO PERIOREITAPUO

\begin{tabular}{|c|c|c|c|c|}
\hline & AUSENTE & LEVE & MODERADO & SEVERO \\
\hline $\begin{array}{l}\text { suncos } \\
\text { ouseunes }\end{array}$ & 0 & 1 & 2 & 3 \\
\hline CELASCNDAS & 0 & 1 & 2 & 3 \\
\hline REL PARYADO BUP & 0 & 1 & 2 & 3 \\
\hline nolen arases & 0 & 1 & 2 & 3 \\
\hline $\begin{array}{l}\text { ARAuOAs } \\
\text { PEnoBitahias }\end{array}$ & 0 & 1 & 2 & 3 \\
\hline TEh.Tmougm & 0 & 1 & 2 & 3 \\
\hline
\end{tabular}

Tabla I.

oírle atentamente mientras analizamos sus rasgos faciales y planificamos las posibles modificaciones o mejorías que podríamos ofrecerle.

Una vez aclarado por parte del paciente cuáles son sus expectativas, y después de un examen físico donde evaluamos distintos parámetros como : tipo de piel, elasticidad de la piel, grado de flacidez, presencia de manchas, arrugas, posición de las cejas, blefarocalasia, bolsas palpebrales, surco de la ojera, etc., pasamos a plantearle qué posibilidades hay de mejorar su problema y cuáles son las técnicas, quirúrgicas o no, que podríamos ofrecerle.

Si el paciente presenta una importante blefarocalasia, caída de la ceja, surcos glabelares marcados, bolsas inferiores muy prominentes y un surco de la ojera muy acentuado, debemos ser concientes de que las posibilidades de éxito serán muy bajas solo con procedimientos estéticos no invasivos.

En la Tabla I exponemos los parámetros que habitualmente evaluamos en el examen físico de los pacientes, para así establecer el diagnóstico y posteriormente el protocolo de tratamiento no invasivo a realizar.

Cada uno de los parámetros, puede no estar presente, ser leve, moderado o severo, asignándole una puntuación de 0,1, 2 ó 3 puntos respectivamente. Si la suma es superior a 12 puntos, nos habla de que algunos de los parámetros tendrían que ser resueltos de manera quirúrgica. Las cejas muy caídas, una gran redundancia de piel en párpado superior o unas bolsas grasas palpebrales inferiores muy prominentes, solo pueden ser resueltos de manera quirúrgica.

Es repetitivo comentar que cada paciente es distinto y que sus necesidades son diversas; pero siempre que seamos claros en la información de lo que podemos ofrecerle y hasta qué punto se resolverán sus problemas, tendremos menos complicaciones y mayor grado de satisfacción tanto por parte del paciente como del cirujano tratante. 


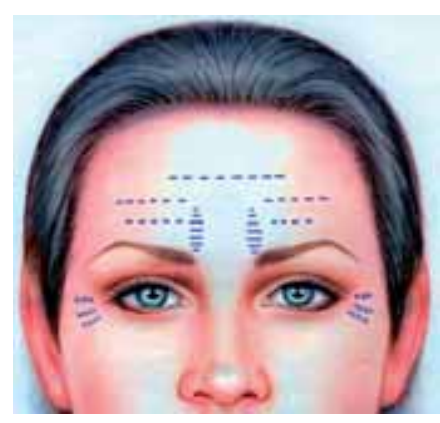

Fig. 1. Infiltración toxina botulínica.

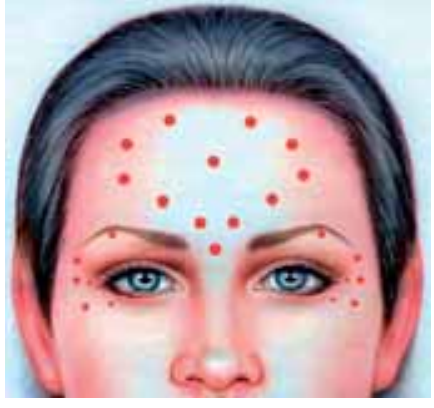

Fig. 2. Infiltración Acido hialurónico

\section{Material y método}

Nuestro grupo de pacientes sometidos a rejuvenecimiento facial no invasivo corresponde a un periodo comprendido entre Junio del 2006 y Enero del 2008, con un total de 109 pacientes de edades comprendidas entre los 28 y 62 años: 78 mujeres y 31 hombres: Todos consultaron para mejorar su rostro y en especial el área peri-orbitaria; de estos 109 pacientes, 83 fueron sometidos a combinaciones de diferentes procedimientos de Medicina Estética en el área facial; 26 pacientes fueron sometidos a cirugía de la mirada (blefaroplastia superior e inferior, cantopexia, elevación de cejas, etc.),de los cuáles, 8 iniciaron previamente tratamiento no invasivo y 10 se sometieron a tratamiento no invasivo después de la cirugía; finalmente 8 , no requirieron otro procedimiento aparte de la cirugía.

Los productos utilizados para el tratamiento médico-estético fueron:

- Toxina botulínica tipo A (Vistabel $\left.{ }^{\circledR}\right)$.

- Acido hialurónico no reticulado y reticulado.

- Vitaminas, oligoelementos, antioxidantes, etc.

- Peeling de alfa-hidroxiácidos.

En relación a la toxina botulínica, empleamos un promedio de 35 a 50 unidades dependiendo del caso; con esto logramos reducir de forma considerable las arrugas glabelares, frontales y periorbitarias, además de elevar y reposicionar la ceja y también disminuir, según el caso, el grado leve o moderado de blefarocalasia superior (2).

La idea de este trabajo no es dar parámetros exactos de la utilización de los diferentes productos, sino explicar nuestra técnica como una propuesta orientativa de tratamiento.

Sabemos que en el caso de arrugas muy profundas a nivel glabelar o periorbitario, podemos complementar el resultado posterior a la aplicación de toxina botulínica con materiales de relleno; en nuestro caso solo utilizamos ácido hialurónico reticulado de mayor o menor densidad según la profundidad de la arruga o surco a tratar (3).
En algunos pacientes aumentamos la elevación de la ceja realizando una columna tensional o hilo químico con un vector ascendente frontotemporal, infiltrando con aplicaciones puntiformes vitaminas mezcladas con acido hialurónico no reticulado (Fig. 1 y 2). En nuestra forma de trabajo, preferimos no mezclar en una misma sesión de trabajo el ácido hilaurónico reticulado y la toxina botulínica, si bien hay especialistas que lo realizan a la vez sin complicaciones aparentes.

Resumiendo, la combinación de toxina botulínica con ácido hialurónico nos permite solucionar en gran parte cuatro de los parámetros fijados para nuestro diagnóstico, a saber, arrugas glabelares, blefarocalasia leve, arrugas periorbitarias y caída de la ceja.

Las bolsas grasas levemente prominentes y el surco de la ojera son dos de los parámetros más difíciles de mejorar, disimular o suavizar mediante procedimientos de Medicina Estética; pero si se es extremadamente cuidadoso y se selecciona adecuadamente el paciente, podemos obtener buenos resultados. Debemos evaluar la calidad de la piel (fina o gruesa), la proyección de las bolsas grasas y lo marcado del surco de la ojera.

Unas bolsas palpebrales muy acentuadas ( 3 puntos), realmente no podrán mejorar con esta técnica, sin embargo el surco nasoyugal acentuado tiene una buena solución con este procedimiento.

Nuestro protocolo de trabajo de este área tiene como objetivo una bioestimulación de la piel para lograr una reestructuración cutánea que aporte un leve aumento de volumen, suavizando el surco que confiere siempre al paciente un aspecto de cansancio y fatiga.

Utilizamos combinaciones de acido hialurónico no reticulado ( A.H.N.R) y vitaminas; las proporciones utilizadas son:

Protocolo A- vitaminas $5 \mathrm{cc}$ más . A.H.N.R. 0,5cc. Protocolo B- vitaminas $5 \mathrm{cc}$ más A.H.N.R. 1cc. Protocolo C-vitaminas $5 \mathrm{cc}$ más A.H.N.R. 1,5cc. Protocolo D- vitaminas $5 \mathrm{cc}$ más A.H.N.R. 2cc.

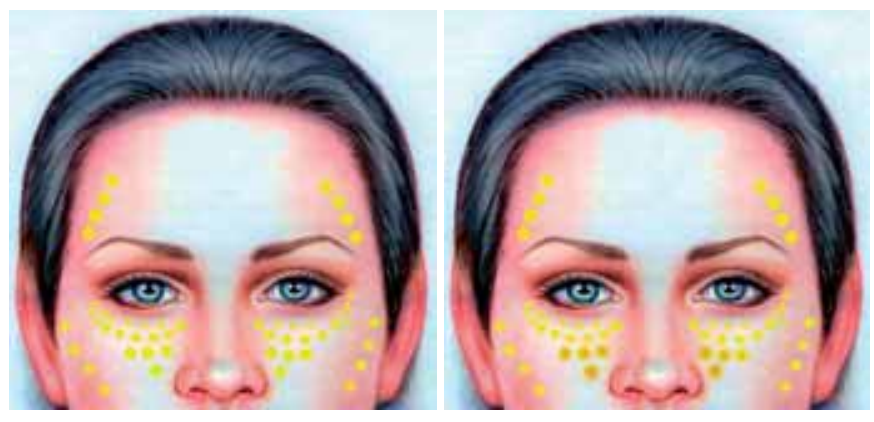

Fig. 3. Infiltración de Protocolos Fig. 4. Infiltración de Protocolos de Vitaminas+A.H.N.R 
El uso de cada uno de estas combinaciones dependerá del daño de la piel y de la profundidad del surco a tratar. Siempre iniciamos nuestra infiltraciones en sentido caudocefálico (de abajo hacia arriba), tanto en cantidad de volumen como en concentración de la solución (A, B, C, o D). El número de sesiones es variable, pero puede ir de 2 a 3 sesiones en un caso leve, a 5 ó 6 sesiones en casos más severos, con un mantenimiento cada 4 a 6 meses. (Fig. 3 y 4).

\section{Resultados}

Presentamos tres casos representativos de los resultados que pueden obtenerse con la adecuada combinación de técnicas de Medicina Estética y explicamos el procedimiento realizado, los materiales utilizados, las cantidades de productos empleadas, los protocolos y el número de sesiones necesarias para resolver cada caso.

Caso1: Mujer de 54 años, que había sido sometida 4 años a una blefaroplastia. En ella podemos evidenciar un surco de la ojera acentuado, con algo de depresión o hundimiento producto de una excesiva reseccion de bolsas grasas en la blefaroplastia previa. El área glabelar y las arrugas periorbitaias se hacían bastante notorias con la gesticulación o mímica facial. Por ultimo, el tercio externo supraorbitario ( próximo a la cola de la ceja) se apreciaba deprimido o esqueletizado.

Previa firma del consentimiento informado y toma de fotografías en diferentes proyecciones del área a tratar, procedimos a realizar el siguiente esquema de trabajo (Fig.5); en primer lugar infiltramos 50 uds. de Vistabel con un esquema bastante similar al que se presenta en la Fig.1, y 2cc de acido hialuronico reticulado, con la distribución aproximada de la señalada en la Fig.2 (4). En cuanto al tratamiento del surco de la ojera y del área palpebral inferior, fueron necesarias 3 sesiones de infiltraciones de A.H.N.R. + Complejo de vitaminas, aminoácidos, etc., utilizados en las combinaciones de los protocolos $\mathrm{B}$ y $\mathrm{C}$ antes mencionados. Infiltramos el protocolo $\mathrm{C}$ en el área más caudal o inferior del surco naso yugal ( $1 / 2 \mathrm{cc}$. aproximadamente en cada lado) y el protocolo $\mathrm{B}$ en el área más próxima al parpado inferior ( $1 / 2 \mathrm{cc}$. aproximadamente en cada lado), siempre infiltrando en pequeños abones, con la consiguiente redistribución digital del material infiltrado. El esquema de aplicación se puede apreciar en las Fig.3 y Fig.4. Siempre usamos para este procedimiento agujas $30 \mathrm{G}$. de 4 $\mathrm{mm}$, y jeringas de 1cc. El intervalo entre sesión fue de entre 10 a 15 días, obteniéndose un resultado satisfac-

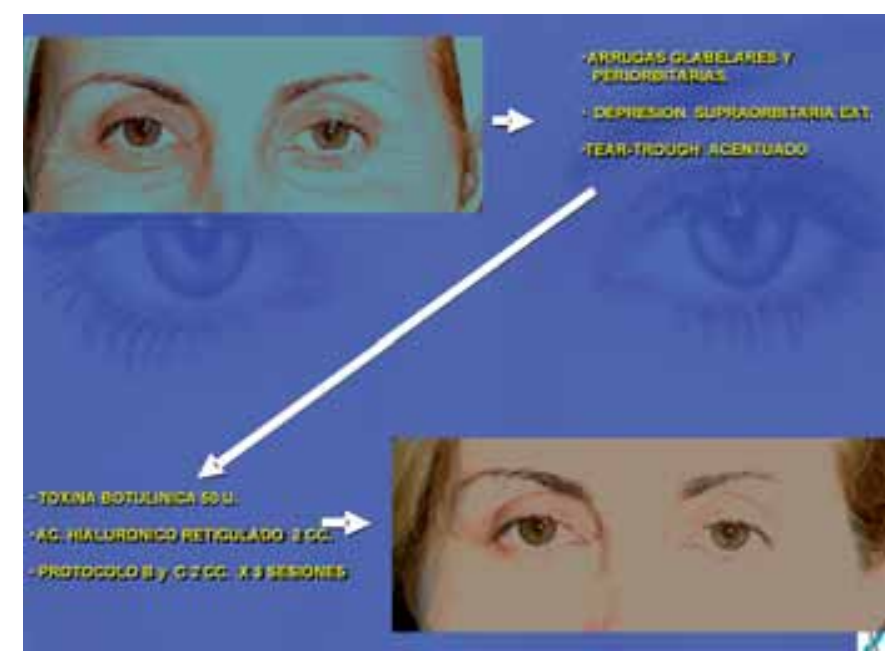

Fig. 5. Esquema de trabajo Casol

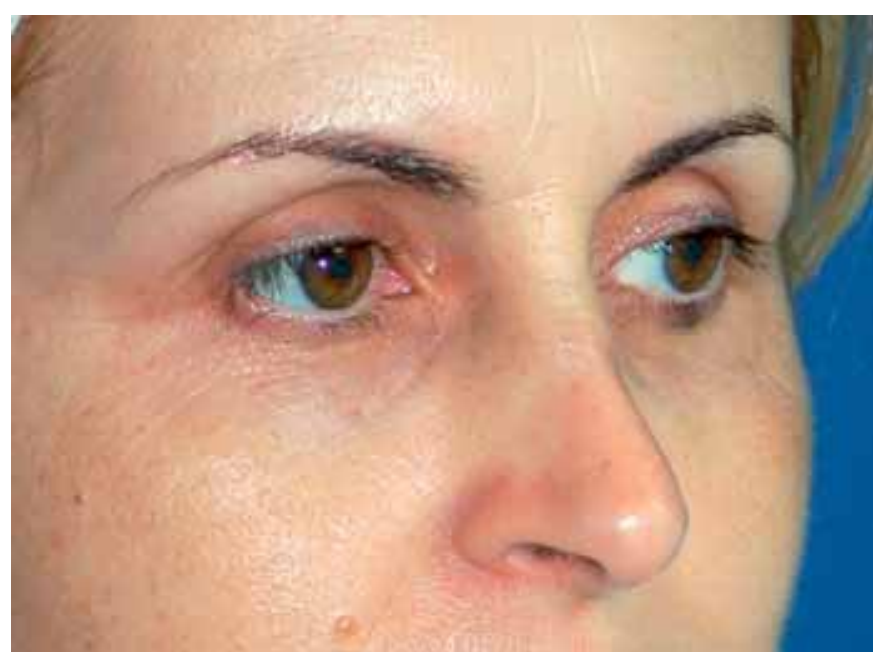

Fig. 6. Caso 1 -Pretratamiento

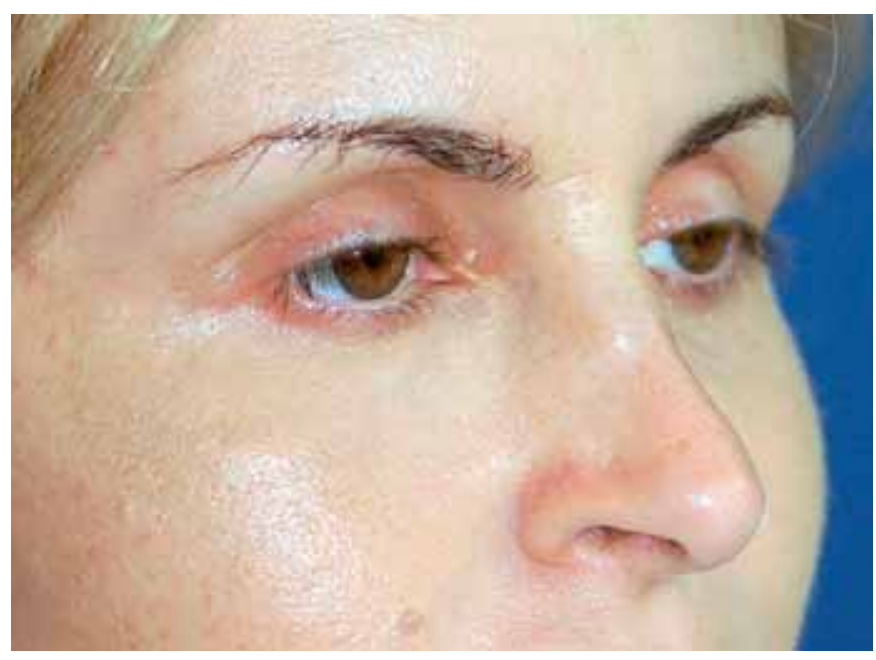

Fig. 7. Caso1 - 3 meses después

torio al cabo de aproximadamente 40 dias y manteniéndose los resultados durante 5 meses. Posteriormente se siguieron los protocolos de mantenimiento necesario (Fig.6 y 7). 


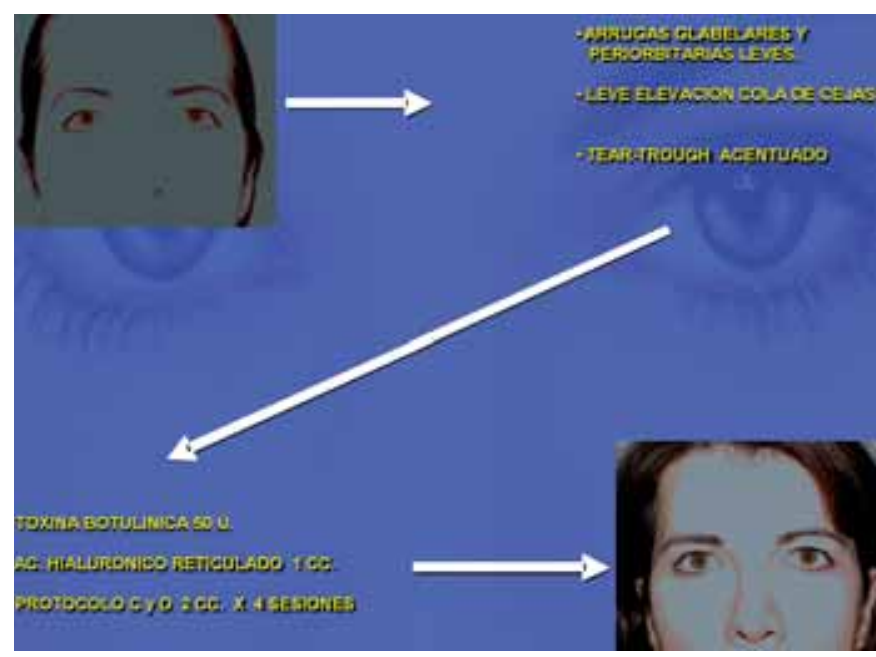

Fig. 8. Esquema de trabajo Caso2

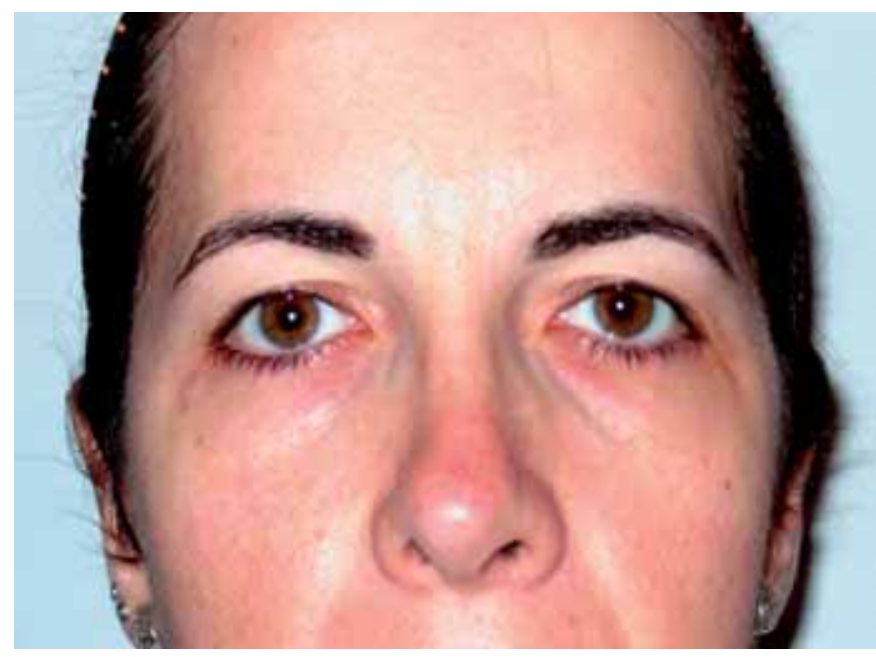

Fig. 9. Caso 2 -Pretratamiento

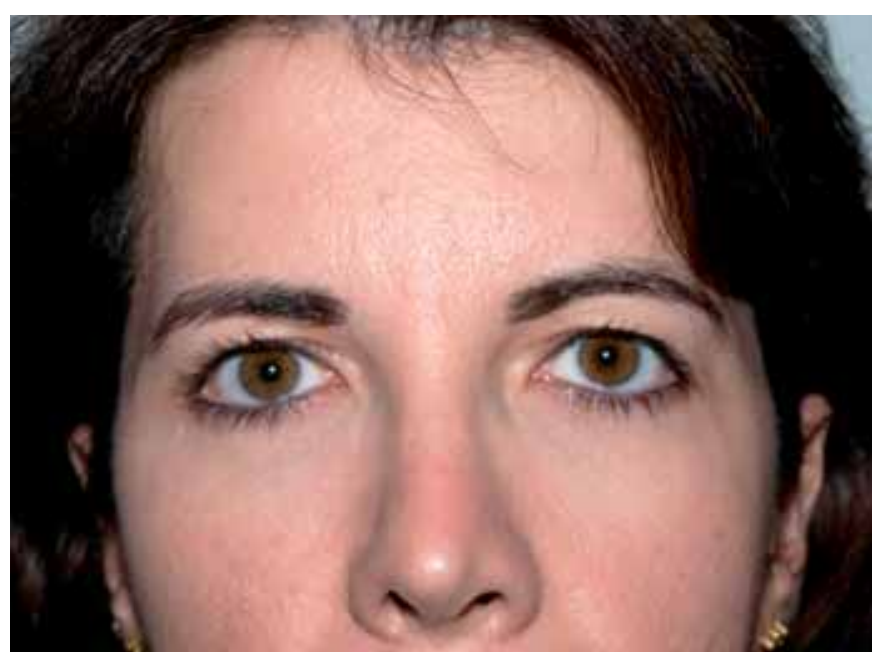

Fig. 10. Caso2: 6 meses después

Caso 2: Mujer de 35 años cuyo problema básico radicaba en lo acentuado del surco nasoyugal, que le hacía presentar siempre un aspecto de cansancio y tristeza. Planificamos el esquema de trabajo como señalamos el la Fig. 8, e iniciamos con la infiltración de 50 uds. de toxina botulínica en el tercio superior facial, haciendo énfasis en elevar la cola de la ceja y $1 \mathrm{cc}$ de acido hialuronico reticulado para finas líneas en el área periorbitaria. En la zona palpebral inferior y surco de la ojera empleamos los protocolos C y D de vitaminas y A.H.N.R. En el área más deprimida y caudal usamos $0,5 \mathrm{cc}$ del protocolo $\mathrm{D}$ para cada lado; y 0,5 del protocolo $\mathrm{C}$ para la zona más alta o próxima al parpado inferior, con la consiguiente redistribución digital de la infiltración. El intervalo de aplicación se realizo cada 15 días durante 4 sesiones consecutivas, con un resultado totalmente satisfactorio y una permanencia de 6 meses. La paciente siguió con el protocolo de mantenimiento de este área, cada 4 a 6 meses según las necesidades (Fig. 9 y 10).

Caso 3: El último caso es el de una mujer de 47 años (Fig.11), cuya particularidad está en que rozaba el límite quirúrgico, pues presentaba unas bolsas grasas moderadas y excedente de piel en parpado inferior. Realizamos infiltración de toxina botulínica, ácido hialuronico para finas líneas en tercio superior facial y por ultimo aplicamos protocolos A y B de vitaminas, aminoácidos, etc. + A.H.N.R para suavizar y dar mayor tono al excedente de piel del párpado inferior, con un resultado apropiado que podemos ver en las Fig.12 y 13.

Si bien este trabajo se centra en el área periorbitaria, realmente pensamos que es la correcta evaluación, planificación y el adecuado uso de cada producto en cada uno de los tercios faciales (superior, medio e inferior) lo que proporciona muy buenos resultados con un alto porcentaje de satisfacción por parte del paciente; esto hace que, entre otras cosas, estos sean además consecuentes con los tratamientos de mantenimiento, pues desean mantener el efecto conseguido tras los resultados del tratamiento inicial.

De los 109 pacientes incluidos en el estudio, 8 fueron resueltos exclusivamente con cirugía, 18 combinaron técnicas quirúrgicas y de Medicina Estética y en 83 se realizaron combinaciones de diferentes procedimientos. Obtuvimos un $94 \%$ de satisfacción en estos pacientes, pues solo 4 abandonaron el tratamiento después de la primera sesión. El promedio de sesiones empleadas fue de 4 por paciente, con una separación entre cada sesión de entre 15 a 20 dias y una sesión de mantenimiento cada 3 a 4 meses para bioestimulación y de 6 a 8 meses para la aplicación de toxina botulínica e infiltración de acido hialurónico.

La evaluación de los resultados, tanto por parte de los pacientes como por el grupo medico, tiene un gran componente de subjetividad, pues la mejoría en 


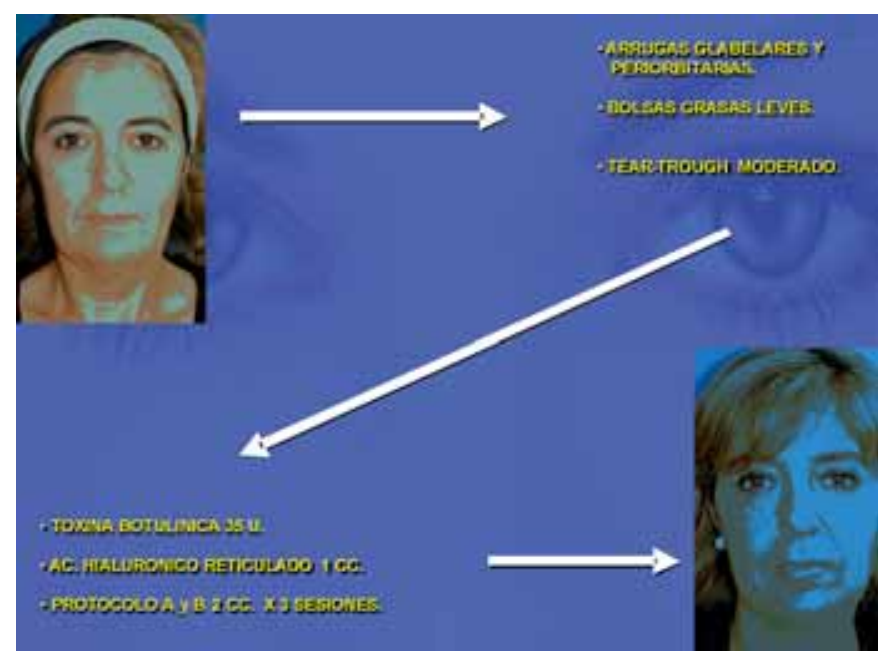

Fig. 11. Esquema de trabajo Caso 3

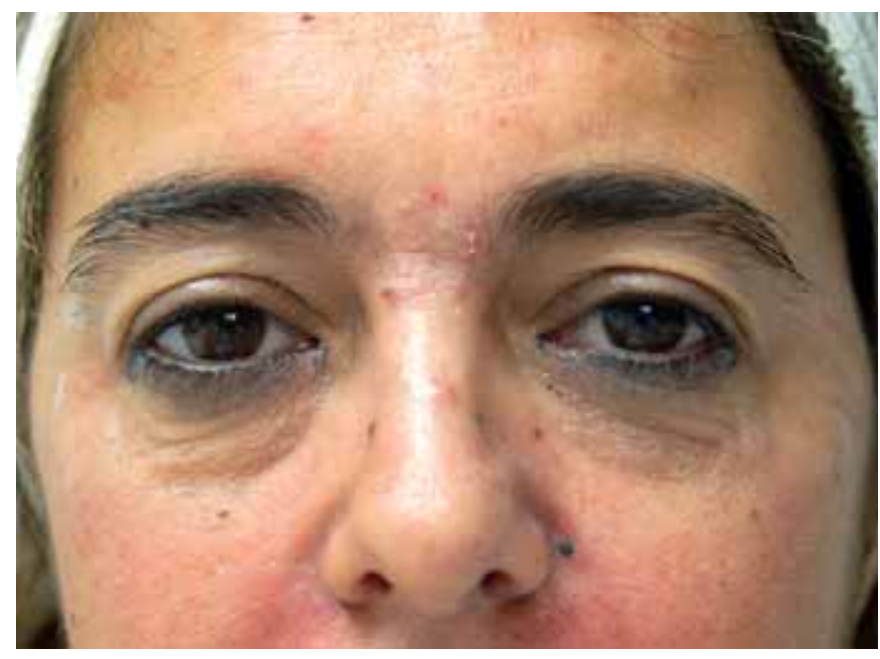

Fig. 12. Caso 3 - Pretratamiento

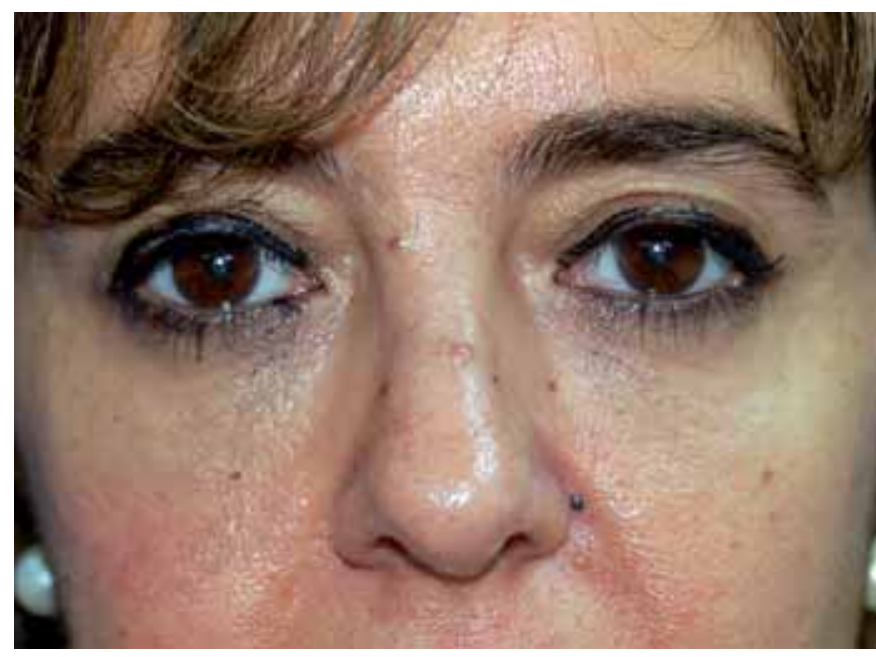

Fig. 13. Caso 3: 4 meses después

la luminosidad de la piel, disminución de arrugas, disminución o reducción del surco de la ojera, son cualitativos y de difícil medición, salvo por la comparación de la iconografía conservada de cada paciente.

\section{Discusión}

Hemos asistido a un cambio en las necesidades y peticiones por parte de los pacientes. Hasta hace 4 a 5 años los de edad madura (40 a 60 años),llegaban a la consulta solicitando que deseaban verse como 10 años atrás; sin embargo hoy en día acuden pacientes más jóvenes (25 a 35 años) que nos plantean lo siguiente: "me veo bien y joven; deseo seguir viéndome igual de bien y evitar los pequeños cambios que deja en el rostro el paso del tiempo" (Fig. 14). Una alternativa para este grupo de pacientes, son las técnicas y protocolos que hemos descrito en este trabajo.

Sabemos lo importante del tratamiento integral del paciente, la adecuada alimentación, administración de antioxidantes por vía sistémica, la indicación de un adecuado programa de ejercicio y los controles en el equilibrio hormonal. Sin embargo, consideramos que esto debe ser llevado a cabo por un equipo multidisciplinario, donde nosotros solo formamos una parte; por supuesto, siempre manteniendo la comunicación con el equipo medico, tanto en la evaluación, diagnostico, tratamiento y seguimiento del paciente, dentro de las pautas de los tratamientos Antienvejecimiento (5).

Con la toxina botulínica logramos atenuar y suavizar las arrugas dinámicas del tercio superior facial, pero igualmente nos permite la posibilidad de modificar o mejorar la posición o ubicación de estructuras como la ceja y canto externo del ojo, proporcionando una mirada más viva, basándonos siempre en el concepto de relajación músculos agonistas -antagonistas.

La corrección del surco de la ojera ha proporcionado un alto grado de satisfacción en nuestros pacientes, al igual que a nuestro equipo de trabajo, pues siendo poco invasivos, evitamos riesgos que pueden presentarse en la corrección de esta área con otros procedimientos y logramos eliminar el aspecto de cansancio y agotamiento que provoca este surco. Recordamos que se han descrito varios procedimientos para la mejoría de esta zona; entre ellos la infiltración profunda de ácido hialurónico, describiéndose como complicación algún caso de embolismo y de consecuente ceguera (6). También la colocación de prótesis de Medpore ${ }^{\circledR}$, pudiéndose observar posteriormente una sobrecorrección del surco nasoyugal. Con los procedimientos de Lipofilling o Técnica de Coleman , probablemente por lo fino de la piel de esta zona, se han descrito casos de quistes o granulomas, evidentes tanto a la inspección como a la palpación (7). 


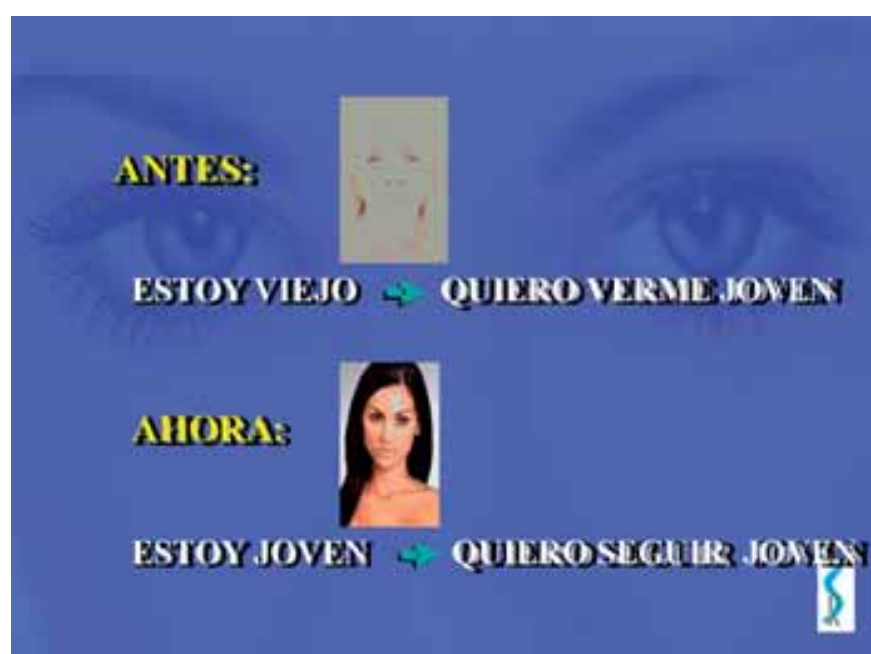

Fig. 14. Evolución del tipo de pacientes que acuden a la consulta.

Con la infiltración de vitaminas+ A.H.N.R en los diferentes protocolos descritos, logramos una bioestimulacion y reestructuración cutáneas, además del efecto local de atracción de líquido e hidratación de la zona, con un efecto voluminizador que permite la corrección inmediata del surco y que a su vez estimula la producción endógena de acido hialurónico y colágeno local mejorando a mediano y largo plazo esta zona, tan delicada de manejar (8).

\section{Conclusiones}

Podemos lograr resultados estéticos satisfactorios en la región periorbitaria con un adecuado conocimiento de la Anatomía y proporciones de este área.

Es necesaria la correcta evaluación del paciente e informar al mismo de las posibilidades de mejoría que pueden lograrse; un buen diagnostico nos llevará a una adecuada elección del protocolo de trabajo y a lograr unos resultados adecuados.

Los diferentes procedimientos que utilizamos, como infiltración de toxina botulínica, acido hialurónico reticulado y la combinación de nutrientes, vita- minas y acido hialurónico no reticulado, protocolizados de forma adecuada, nos permiten mejorar y rejuvenecer el área periorbitaria.

En el área del surco de la ojera es donde logramos los resultados más satisfactorios tanto para el paciente como para el equipo de trabajo, pues con procedimientos relativamente sencillos y seguros, proporcionamos un aspecto más relajado y juvenil.

Debemos conciencienciar al paciente de la necesidad de un seguimiento y de un mantenimiento del tratamiento, para mantener en el tiempo los resultados obtenidos.

\section{Dirección del autor}

Dr. Juan L. Muñoz del Olmo

Servicio de Cirugía Plástica y Estética, Nuevo Hosital Quirón

Plaza Alonso Comín 5-7, $4^{\circ} \mathrm{C} 405$

Barcelona. España

e-mail: delolmojl@hotmail.com

Bibliografía

1. Ramirez, O. M., Novo Torres, A., Volpe, Ch. R.: "El ojo hermoso". Cir. plást. iberolatinoam.2007;33 (2):79.

2. De Maio, M., Wahl, G.: " Expert Approaches to Using Botulinim Toxins. Series” Editor: Dr. Phillip Levy. Nov.2006 (5).

3. Carruthers J, Carruthers A.: "Restylane SubQ in Aesthetic Facial Contouring". Aesthetic Surgery (Supplement) 26. January 2006

4. Zbili, M.: "Personal experience in the filling of wrinkles and remodelling lips with Juvederm". J. de Med. Esth. Et Ch. Derm.Dec.2002, vol XLIII, 98

5. Serra JM: “ Protocolo médico en antienvejecimiento. La importancia de los radicales libres". Cir. plást. iberolatinoam. 2003, 29 (2): 149 .

6. Consensus Recommendations for Soft-Tissue Augmentation with Nonanimal Stabilized Hyaluronic Acid (Restylane). Plast. Reconstr. Surg. ( Supplement). 117. March 2006.

7. Serra JL, Del Olmo JL: "Uso de Factores de Crecimiento Plaquetario unidos a injerto de grasa en lipofilling facial en ritidectomía". Cir. plást. iberolatinoam. 2006 (32) 3: 191.

8. Zbili, M.: «Classification des lèvres et protocoles thérapeutiques: expérience personnelle avec le Juvederm». Med. Esth.et Chir. Derm. 2004, Vol XXXI:121. 